\title{
Flow and flow-induced vibration of a square array of cylinders in steady currents
}

Ming Zhao ${ }^{1, *}$, Liang Cheng ${ }^{\mathrm{b}}$, Hongwei An ${ }^{\mathrm{b}}$, Feifei Tong ${ }^{\mathrm{b}}$

${ }^{a}$ School of Computing, Engineering and Mathematics, University of Western Sydney, Locked Bag 1797, Penrith, NSW 2751, Australia

b School of Civil and Resource Engineering, The University of Western Australia, 35 Stirling Highway, Crawley, WA 6009

* Corresponding author. Email: m.zhao@uws.edu.au, Tel: +61 247360085

\section{ABSTRACT}

Flow and flow-induced vibration of a square array of cylinders are investigated by two-dimensional numerical simulations. Flow past 36 cylinders in an inline arranged square array and 33 cylinders in a staggered arranged square array is firstly simulated, for $\operatorname{Re}=100$ and the spacing ratios of $L / D=1.5$, $2,3,4,5$. Only one vortex street is observed in the wake of the cylinder array when the spacing ratio is 1.5 in the inline arrangement and 1.5 and 2 in the staggered arrangement, indicating that the critical spacing ratio for the single-vortex street mode in the staggered arrangement is higher than that in the inline arrangement. The vortex shedding from the cylinders is suppressed at $L / D=3$ for both inline and staggered arrangements. Vortex shedding from each individual cylinder is observed when $L / D=4$. Flow-induced vibration of 36 cylinders in an inline square arrangement is studied for a constant Reynolds number of 100, two spacing ratios of 2 and 5, a constant mass ratio of 2.5 and a wide range of reduced velocities. It is found that for a spacing ratio of 2 , the vibration of the cylinders in the four downstream columns does not start until the reduced velocity exceeds 4.5 . The vibration of the cylinders progresses downstream with increasing reduced velocity. For a spacing ratio of 5, the vibrations of the cylinders in the most upstream column are similar to that of a single cylinder. The vibration amplitudes of the downstream cylinders peak at higher reduced velocities than that of a single cylinder. The maximum possible response amplitudes occur at the most downstream cylinders. 


\section{Introduction}

Flow through a group of cylinders is relevant to many engineering structures such as heat exchangers, offshore riser pipes and nuclear reactors. Flow characteristics around multiple cylinders depend on the arrangement of the cylinders and the spacing among the cylinders. Flow past two cylinders has been the topic of many studies because it provides very fundamental mechanisms of flow interference (Sumner, 2010; Tong et al., 2015). When two side-by-side cylinders are in a fluid flow, only one vortex street is formed in the wake of the cylinders if the spacing ratio is less than 2.2 (Bearman and Wadcock, 1973; Williamson, 1985; Kim and Durbin, 1988). The wakes from the two cylinders are found to synchronize for a certain range of spacing ratios (Williamson, 1985). Alam et al. (2003) reported that the gap flow was biased towards one cylinder if the spacing ratio between two side-by-side cylinders was less than 2.2. If two cylinders are in a tandem arrangement, the vortex shedding occurs only from the downstream cylinder if the spacing ratio is less than about 3 to 3.5, depending on the Reynolds number (Meneghini et al., 2001; Mizushima and Suehiro, 2005; Tasaka et al., 2006). The Reynolds number is defined as $\operatorname{Re}=U D / D$, where $U$ is the free stream velocity, $D$ is the cylinder diameter and $v$ is the kinematic viscosity.

Flow around four cylinders in a square arrangement was also investigated extensively. Sayers (1988) measured the drag and lift coefficients on each individual cylinder in a group of four in a square arrangement. The forces were found to be greatly influenced by the orientation of the cylinder group. The experimental study by Sayers (1990) about flow past four cylinders, which was focused on the vortex shedding frequency, showed that at certain spacing ratios and flow inclination angles asymmetrical vortex shedding took place. Lam and Lo (1992) conducted flow visualizations of steady flow past four cylinders in an inline square arrangement at a Reynolds number of 2100. It was found that the vortex shedding of the upstream cylinders was suppressed as the spacing ratio became less than 3.94D. Bistable flow in the wake of the downstream cylinders was observed when the 
spacing ratio was less than 1.70. Lam and Fang (1995) and Lam et al. (2003a; 2003b) measured the pressure distributions and the forces of four cylinders in a square arrangement and reported that the flow pattern had significant effects on the pressure distributions and the force coefficients. By visualizing the flow pattern using a digital particle image velocimetry (DPIV) and also numerical simulations, Lam and Zou (2009, 2010) observed several distinct flow patterns depending on the spacing ratio. Farrant et al. (2000) simulated flow past four cylinders in an inline square arrangement numerically and reported an in-phase vortex shedding mode at small spacing ratios and an anti-phase vortex shedding mode at large spacing ratios. Han et al. (2013) found three types of flow patterns for flow past four cylinders in a square arrangement at $\mathrm{Re}=200$. The wake flow was found to be similar to a single-bluff body flow pattern when the spacing ratio is small. The wiggling shielding wake was observed at an intermediate spacing ratio of 1.6. Four vortex streets were observed when the spacing ratio was 3.5 - 4.0. By conducting three-dimensional numerical simulations, Tong et al. (2014) found that the flow past four cylinders in an inline arrangement at a constant spacing ratio of 2 and Reynolds numbers in the range between 100 and 500 can be classified into four flow regimes and change in the flow regime has a significant effect on the force.

With increasing number of cylinders $(n)$ in a cylinder system, flow around a cylinder system resembles to flow through a porous medium (Nicolle and Eames, 2011). Thus the forces induced on the cylinders and the flow field through and around the cylinder group are highly related to the void fraction. By conducting wind tunnel tests of flow about an array of up to $9 \times 9$ cylinders, Ball and Hall (1980) reported that the drag force on the cylinders was dependent on the flow incident angle. By conducting numerical simulations, Nicolle and Eames (2011) studied the effect of void fraction on flow past a circular array of cylinders ranging from 7 to 133 with a fixed Reynolds number of 100 (based on the individual cylinder diameter) and fixed array diameter of $21 D$. The void fraction was found to be inversely proportional to the number of cylinders in the array. The flow in the wake of the cylinder array was found to be similar to that in the wake of an isolated body for sufficiently 
small void fractions. The wake flow was found to be stable and the force on a cylinder array was found to be steady at moderate void fractions. For high void fractions, the flow interaction was found to be weak and force characteristics on each cylinder are similar to those of an isolated cylinder. Yu et al. (2013) simulated flow through a circular array of cylinders in the Reynolds number range of 100-2000 using a hybrid RANS/LES turbulence model. The drag coefficient was found to increase with the solid volume fraction, but decrease with increasing Reynolds number. It was also found that the drag coefficient was independent on the diameter of the cylinder array when the solid volume fraction and the Reynolds number were kept constant. Sweeney and Meskell (2003) used a fast discrete vortex method to simulate flow past cylinder arrays at a Reynolds number of 2200. Their numerical result of Strouhal number was close to the published data and the vortex shedding pattern qualitatively agreed with the flow visualization. Based on the definitions of Eulerian and Lagrangian mean velocities and their decompositions into near and far field components, Ermes et al. (2004) developed a numerical model for analysing inviscid flow through groups of bodies. Dehkordi and Jafari (2009) simulated flow past groups of circular tubes in a square and staggered arrangements. Two different flow patterns were observed by Dehkordi and Jafari (2009), namely the pattern through the bundles and the pattern around the bundles. Kevlahan and Wadsley (2005) studied the suppression of the three-dimensionality for flow past a square array of 9 tubes with a spacing ratio of 1.5. It was found that the tube motion did not suppress the three-dimensional instability, but did increase the spanwise correlation. Longatte et al. (2003) reported that the disappearance of the wake flow for a cylinder array enables the simplified potential flow model to be applied to predict the flow approximately.

Flow-induced vibration of cylinders is also of engineering significance and has been well documented in literature. When a fluid flow passes an elastically mounted cylinder, the synchronization (or lock-in) between the vortex shedding frequency and the vibration frequency occurs over a range of reduced velocities and the range of reduced velocity for lock-in is dependent 
on the mass ratio of the system and the Reynolds number (Feng, 1968; Govardhan and Williamson, 2000; Jauvtis and Williamson, 2004; Khalak and Williamson, 1996, 1997, 1999). The reduced velocity is defined as $V_{\mathrm{r}}=U / f_{\mathrm{n}} D$, with $f_{\mathrm{n}}$ the natural frequency of the cylinder. The mass ratio is defined as the ratio of the cylinder mass to the displaced mass of the fluid. In order to find fundamental mechanisms of vortex-induced vibrations (VIV), many numerical studies were conducted at very low Reynolds numbers in the laminar flow regime (Anagnostopoulos and Bearman, 1992; Mittal and Kumar, 1999; Singh and Mittal, 2005; Prasanth et al., 2006; Leontini et al., 2006). The lock-in regimes of the reduced velocity at low Reynolds numbers were found to be narrower than those at high Reynolds numbers. Guilmineau and Queutey, (2004), Pan et al. (2007), Kataoka, (2008) and Zhao and Cheng (2011) simulated VIV of a cylinder in turbulent flows by solving the Reynolds-Averaged Navier-Stokes (RANS) equations. The RANS equations were found to give reasonably good predictions of response amplitude and frequency of the cylinder.

Recent studies of VIV of multiple cylinders were mainly focused on two-cylinder systems. Fontaine et al. (2006) classified the response of two riser pipes in a tandem arrangement in two categories: Wake Induced Oscillation (WIO) and Vortex Induced Vibration (VIV). WIO (also referred to as galloping) is known to be a self-limited motion with a maximum order of $1 D$ for a single riser (Ruscheweyh , 1983; Brika and Laneville, 1999). Bokaian and Geoola (1984) found that the vibration of an elastically mounted circular cylinder in the wake of a fixed upstream cylinder could be a VIV, a galloping or a combination of VIV and galloping, depending on the gap between the two cylinders. Numerical studies of VIV of two cylinders in tandem were mainly conducted at relatively low Reynolds numbers and gaps between the two cylinders of $5 D$ or $5.5 D$ (Mittal and Kumar, 2001, 2004; Bao et al., 2011). The studies of VIV of two side-by-side cylinders in fluid flow are fewer than those of two tandem cylinders. Huera-Huarte and Gharib (2011) investigated vortexinduced vibration of two flexible cylinders in side-by-side and tandem arrangements. The interference between the two cylinders was found to be very weak if the centre-to-centre gap 
exceeded 3.5D. Rahmanian et al. (2012), Zang et al. (2013) and Zhao and Yan (2013) investigated VIV of two cylinders of different diameters close to each other and found the interference between the two cylinders has significant effect on VIV. The studies of VIV of four cylinders are even fewer than that of two cylinders. Zhao and Cheng (2012) simulated vortex-induced vibration of four cylinders in a square arrangement numerically at a spacing ratio of 3 by solving the ReynoldsAveraged Navier-Stokes equations. The lock-in regime of the reduced velocity was found to be widened at small alignment angles ( $0^{\circ}$ alignment angle corresponding to the inline arrangement) and the response amplitude was found to be the weakest at an alignment angle of $45^{\circ}$ (diamond arrangement).

The studies of flow-induced vibration of an array of cylinders are limited. When a cylinder in an array of cylinders is flexible, the change of the fluid forces can induce instability if the energy input by the fluid force exceeds the energy due to damping and leads to the vibration of the cylinder (Blevins, 2001). Price et al. (1995) conducted flow visualization experiments for the interstitial cross-flow through a parallel triangular array of cylinders with a spacing ratio of 1.375 and a rotated square array with a spacing ratio of 1.5. No vortex shedding was found for the parallel triangular array. However, if one of the third or fifth row cylinders was replaced by a flexible cylinder, large amplitude vibration was observed. Kevalahan (2011) presented results of the potential flow and twodimensional Navier-Stokes calculations and found that vortex dynamics is the dominant factor determining the vibrational stability of inline tube arrays.

This paper is focused on flow and flow-induced vibration of a cylinder array in a square arrangement at low Reynolds number. The investigation is carried out through numerical tests by solving the two-dimensional incompressible Navier-Stokes equations. A low Reynolds number of 100 based on individual cylinder diameter and a low mass ratio of 2.5 are chosen in the simulations. Studies of VIV of cylindrical structures at low mass ratios are relevant to the response of the offshore structures such as pipelines, riser pipes in water flow. It is well know that the flow in the wake of a 
single cylinder becomes three-dimensional if the Reynolds number exceeds about 140-190 (Williamson, 1996). $\mathrm{Re}=100$ is chosen to be the same as that used in previous studies for a cylinder bundle with a large number of cylinders (Nicolle and Eames, 2011; Dehkordi and Jafari, 2009), which is large enough to trigger vortex shedding for an individual member in the array, but considerably small to minimize the three-dimensionality of the flow. A low Reynolds number is considered because simulations for flow past an array of cylinders with large Reynolds numbers in the turbulent flow regimes require tremendous and unaffordable computational time.

Simulations for flow past a square array of stationary cylinders are conducted for an inline arranged array of 36 cylinders and a staggered arranged array of 33 cylinders as shown in Fig. 1. The effects of the spacing ratio on the flow pattern and the force coefficients on the cylinders are discussed. When flow-induced vibrations of an array of the cylinders are simulated, each cylinder is elastically supported and can only vibrate in the cross-flow direction as shown in Fig. 2.
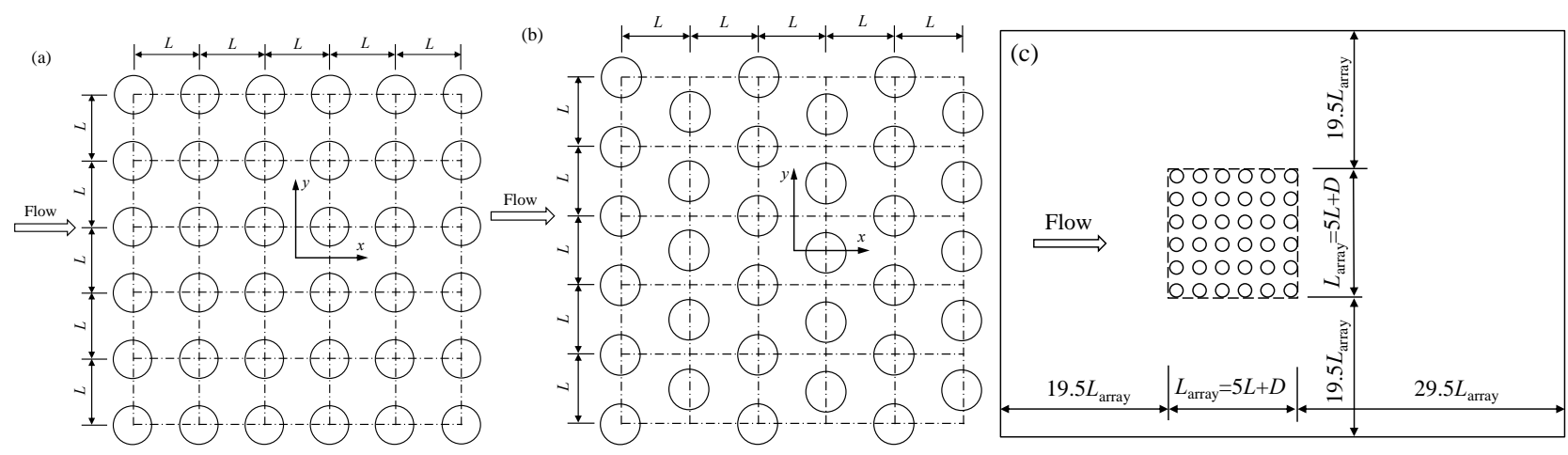

Fig. 1 Inline and staggered configurations of a square array of stationary cylinders in a fluid flow. (a) Inline arrangement; (b) Staggered arrangement; (c) Computational domain. 


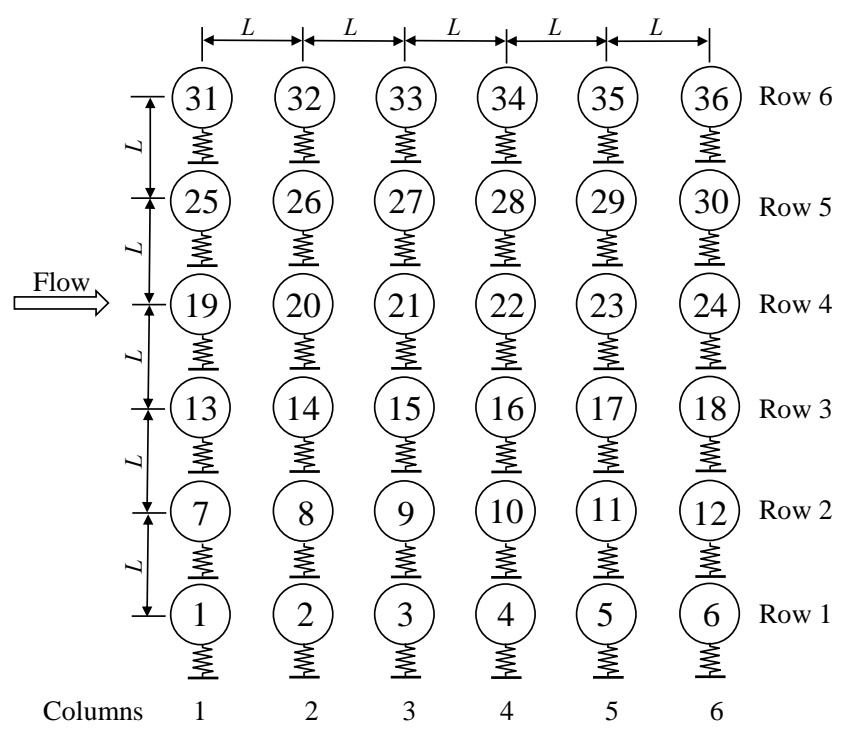

Fig. 2 Sketch of elastically supported an array of cylinders in an inline square arrangement

\section{Numerical method}

A Cartesian coordinate system is defined with its origin at the centre of the cylinder array and the $x$-axis pointing the flow direction. The governing equations for simulating the fluid flow are the incompressible two-dimensional Navier-Stokes (NS) equations. The ALE formulation of the NS equations for incompressible flows are expressed as

$$
\begin{aligned}
& \frac{\partial u_{i}}{\partial t}+\left(u_{j}-u_{j, \text { mesh }}\right) \frac{\partial u_{j}}{\partial x_{j}}+\frac{1}{\rho} \frac{\partial p}{\partial x_{i}}=v \frac{\partial^{2} u_{i}}{\partial x_{j} \partial x_{j}}, \\
& \frac{\partial u_{i}}{\partial x_{i}}=0,
\end{aligned}
$$

where $x_{1}=x$ and $x_{2}=y$ are the Cartesian coordinates in the inline and the transverse directions of the flow, respectively; $u_{i}$ is the fluid velocity component in the $x_{i}$-direction; $u_{i \text {,mesh }}$ is the velocity of the computational mesh, $t$ is the time and $\rho$ is the density of the fluid. In the ALE scheme, the mesh nodes are moved according to the displacements of the cylinders and the velocity $u_{i \text {,mesh }}$ of the movement of the computational mesh is included in the NS equations to account for the effect of the mesh movement. $u_{i \text {,mesh }}=0$ if the cylinders are stationary. The equation of the motion of each cylinder is 


$$
m \frac{\partial^{2} Y}{\partial t^{2}}+c \frac{\partial Y}{\partial t}+k Y=F_{L}
$$

where $Y$ is the displacement of the cylinder in the cross-flow direction, $m$ is the mass of the cylinder, $c$ is the damping constant, $k$ is the spring constant and $F_{L}$ is the lift force of the cylinder. The equations of motion Eq. (3) is solved using the fourth-order Runge-Kutta algorithm. The NS equations are solved by the Petrov-Galerkin finite element method (PG-FEM), which has been successfully used in the simulations of two-dimensional flow-induced vibrations of two cylinders at low Reynolds numbers by Zhao (2013) and Zhao and Yan (2013) and the three-dimensional flowinduced vibration by Zhao and Cheng (2014) and Zhao et al. (2014). For each cylinder array configuration, a rectangular computational domain of a length of $50 L_{\text {array }}$ in the inline direction and a width of $40 L_{\text {array }}$ in the cross-flow direction is used as shown in Fig. 1 (c), where $L_{\text {array }}=5 L+D$ is the boundary length of the whole square cylinder array. The cylinder array is in the middle of the computational domain in the cross-flow direction and $20 L_{\text {array }}$ downstream the inlet boundary. Prasanth and Mittal (2008) found that the blockage only affects the occurrence of the hysteresis in a very narrow reduced velocity regime if it is less than $5 \%$. It is expected that the blockage of $2.5 \%$ in this study will have little effects on flow-induced vibrations.

To impose boundary conditions, the velocity at the inlet boundary is specified according to the reduced velocity. On the surface of each cylinder, the velocity of the fluid is the same as the vibration velocity of the cylinder. At the two side boundaries that are parallel to the flow direction, the velocity component in the cross-flow direction is zero and the gradient of the inline velocity component in the cross-flow direction is zero.

In ALE scheme, the finite element nodes need to be moved after each computational time step according to the updated positions of the cylinders. The governing equation for calculating the displacements of the FEM mesh is (Zhao and Cheng, 2011)

$$
\nabla \cdot\left(\gamma \nabla S_{y}\right)=0
$$


where $S_{y}$ is the displacement of the mesh nodes in the cross-flow direction and $\gamma$ is a parameter that controls the mesh deformation. In order to avoid excessive deformation of the near-wall elements, the parameter $\gamma$ in a finite element is set to be $\gamma=1 / A$, with $A$ the area of the element. Eq. (4) is solved by a Galerkin FEM. The boundary conditions for Eq. (4) are that the displacement of the mesh is the same as the vibration displacement on the cylinder surfaces and zero on the rest of the boundaries. When the cylinders are allowed to vibrate, collision between two cylinders may occur when the spacing ratio is small. If two cylinders collide with each other, the simulation cannot be continued because mesh between them is extremely distorted. The method for treating the collision between two cylinders is the same as the one used in Rahmainian et al. (2014). In the numerical simulations, collision occurs if the gap between two cylinders is sufficiently small $(0.02 D)$. The velocities of the two cylinders after the collision are calculated based on the conservations of energy and momentum. If two cylinders with the same mass and the same volume collide with each other, they exchange their velocities during the collision.

\section{Mesh dependency study}

The present numerical model has been validated against a number of similar flow problems to the one investigated in this study and therefore no further validation of the model is carried out in this study. Zhao (2013) validated the present numerical model against the published data of flow past two cylinders at $\mathrm{Re}=200$ and VIVs of a single cylinder and a two-cylinder system at $m^{*}=2.34$ and $\mathrm{Re}=150$. Zhao et al. (2013) also validated the model against the published data for VIV of a single cylinder at $m^{*}=10, \operatorname{Re}=100$.

Fig. 3 shows two typical meshes for an inline arrangement at $L / D=3$ and the staggered arrangement at $L / D=1.5$. The mesh dependency study is conducted for flow past four cylinders in an inline square arrangement at $\mathrm{Re}=100$. The structure of mesh for flow past four cylinders is similar to that shown in Fig. 3 (a) only with fewer cylinders. For each spacing ratio $L / D$, three meshes are used 
in the simulation. The element number along the surface of each cylinder is 80 for the coarse and normal meshes and 120 for the dense mesh. The minimum mesh sizes in the radial direction of each cylinder are $0.01 D, 0.0025 D$ and $0.001 D$ for the coarse, normal and dense meshes, respectively. The Strouhal number $\left(S_{\mathrm{ti}}\right)$, the mean (averaged over time) drag and lift coefficients $\left(\bar{C}_{\mathrm{Di}}\right.$ and $\left.\bar{C}_{\mathrm{Li}}\right)$, the root mean square (RMS) drag and lift coefficients ( $C_{\mathrm{Di}}^{\prime}$ and $C_{\mathrm{Li}}^{\prime}$ ) of the two bottom cylinders calculated from the three meshes are listed in Table 1. The drag and lift coefficients of an individual cylinder are defined as $C_{\mathrm{Di}}=F_{\mathrm{Di}} /\left(\rho D U^{2} / 2\right)$ and $C_{\mathrm{Li}}=F_{\mathrm{Li}} /\left(\rho D U^{2} / 2\right)$, with $F_{\mathrm{Di}}$ and $F_{\mathrm{Li}}$ are the drag and lift forces of the cylinder, respectively. The Strouhal number of an individual cylinder is defined as $S_{\mathrm{ti}}=f_{\mathrm{s}} D / U$, with $f_{\mathrm{s}}$ being the vortex shedding frequency of the cylinder. It can be seen that the coarse mesh gives better prediction of the Strouhal number than the force coefficients. There is little difference between the results from the normal and dense meshes. In the simulation of flow past a cylinder array, the mesh density inside and around the array is the same as that of the normal mesh used in the mesh dependency study.

(a)

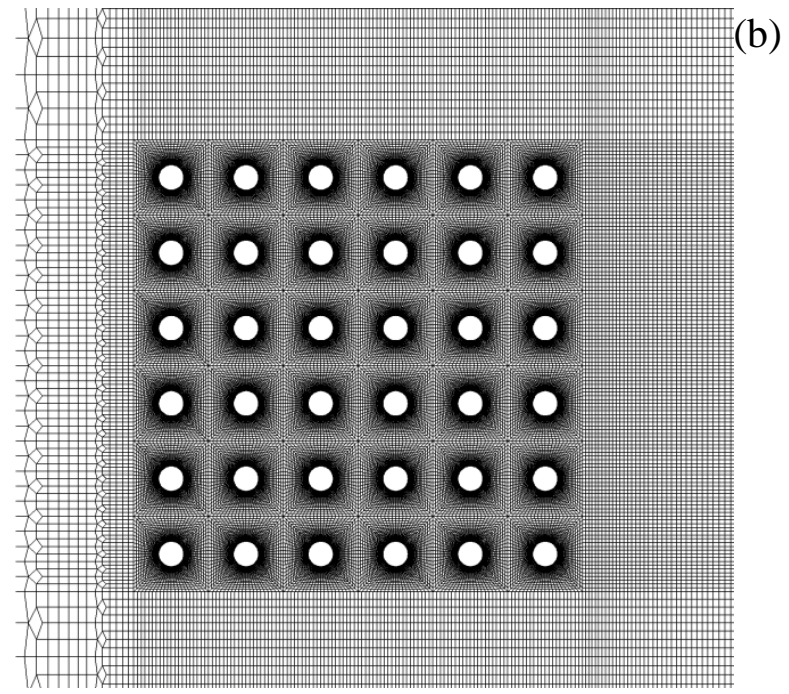

(b)

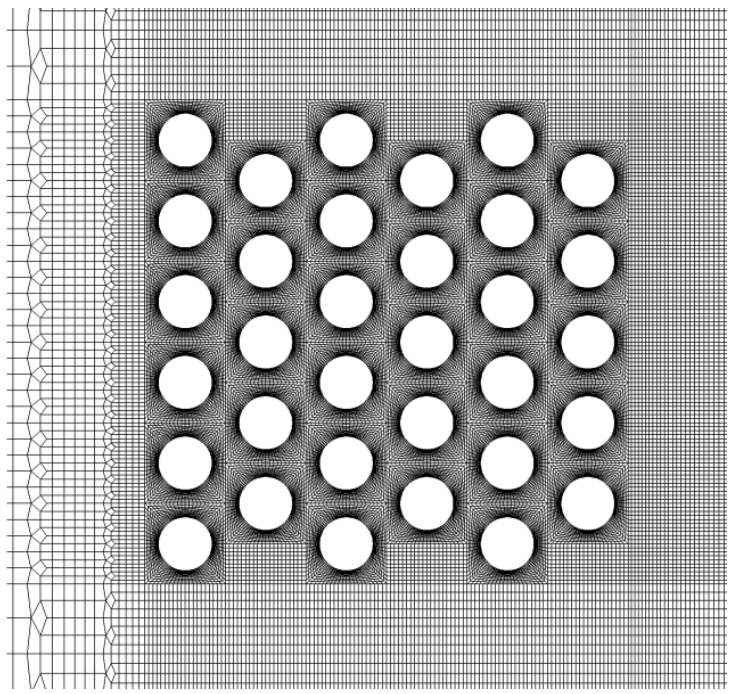

Fig. 3 Computational mesh around the cylinders. (a) $L=3$, Inline arrangement; (b) $L=1.5$, staggered arrangement 
Table 1 Comparison among the Strouhal number and the force coefficients from different meshes

\begin{tabular}{|l|l|l|l|l|l|l|l|l|l|l|l|}
\hline \multirow{2}{*}{$L / D$} & \multirow{2}{*}{ Mesh } & \multicolumn{4}{|l|}{ Upstream bottom cylinder } & \multicolumn{4}{|c|}{ Downstream bottom cylinder } \\
\cline { 3 - 12 } & & $S_{\mathrm{ti}}$ & $\bar{C}_{\mathrm{Di}}$ & $C_{\mathrm{Di}}^{\prime}$ & $\bar{C}_{\mathrm{Li}}$ & $C_{\mathrm{Li}}^{\prime}$ & $S_{\mathrm{ti}}$ & $\bar{C}_{\mathrm{Di}}$ & $C_{\mathrm{Di}}^{\prime}$ & $\bar{C}_{\mathrm{Li}}$ & $C_{\mathrm{Li}}^{\prime}$ \\
\hline 1.5 & coarse & 0.088 & 1.363 & 0.009 & -0.414 & 0.007 & 0.086 & 0.172 & 0.005 & -0.135 & 0.011 \\
\hline 1.5 & normal & 0.086 & 1.412 & 0.002 & -0.436 & 0.004 & 0.088 & 0.217 & 0.009 & -0.142 & 0.013 \\
\hline 1.5 & dense & 0.086 & 1.414 & 0.002 & -0.438 & 0.004 & 0.088 & 0.220 & 0.010 & -0.140 & 0.013 \\
\hline 3 & coarse & 0.136 & 1.314 & 0.006 & -0.146 & 0.078 & 0.136 & 0.281 & 0.094 & -0.074 & 0.432 \\
\hline 3 & normal & 0.137 & 1.343 & 0.005 & -0.150 & 0.065 & 0.137 & 0.265 & 0.088 & -0.082 & 0.403 \\
\hline 3 & dense & 0.137 & 1.345 & 0.005 & -0.152 & 0.063 & 0.137 & 0.260 & 0.086 & -0.083 & 0.401 \\
\hline
\end{tabular}

\section{Flow past a stationary square array of cylinders}

Flow past a stationary square array of cylinders is firstly simulated and the forces and the wake patterns are analysed. The Reynolds number based on the diameter of individual cylinders is fixed at $\mathrm{Re}=100$ in all the simulations. Simulations are conducted for a non-dimensional time $(U t / D)$ of at least 2000. The simulated time for irregular vortex shedding flows is even longer. Fig. 4 shows the vorticity contours for the fully developed flow for $L / D=1.5,2,3,4$ and 5 at the last computational time step. The nondimensional vorticity is defined as $\omega=(\partial v / \partial x-\partial u / \partial y) /(U / D)$. Fig. 5 shows the time histories of the force coefficient for a stationary cylinder array. The drag and lift coefficients on a whole array of the cylinders is defined as $C_{\mathrm{D}}=F_{\mathrm{D}} /\left(n \rho D U^{2} / 2\right)$ and $C_{\mathrm{L}}=F_{\mathrm{L}} /\left(n \rho D U^{2} / 2\right)$, where $F_{\mathrm{D}}$ and $F_{\mathrm{L}}$ are the total drag and lift forces on all the cylinders, respectively and $n$ is the number of the cylinders. 

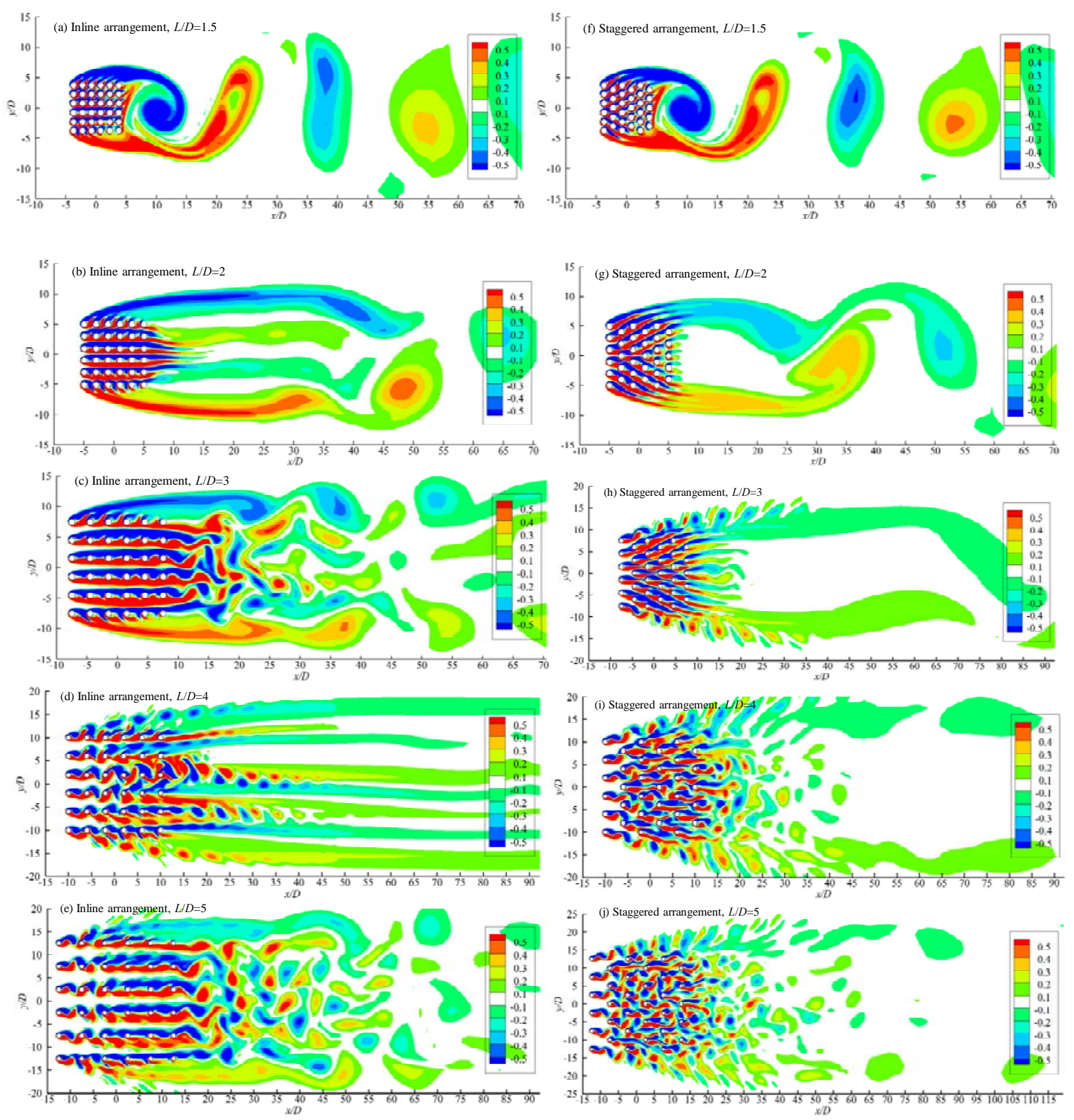

Fig. 4 Contours of the nondimensional vorticity for flow past a fixed cylinder array

Based on the force coefficient in Fig. 5 it can be seen that the flow is not fully developed until $U t / D>1000$ in some cases. When the spacing ratio $L / D=1.5$, the flow through the cylinder array is weak and no vortex shedding happens from individual cylinder (refer to Fig. 4 (a) and (f)). Strong shear layers developed from the top and bottom sides of the cylinder array lead to very strong vortex shedding. Similar to that of a single body, a single vortex street is observed in the wake of the cylinder array with both the inline and the staggered arrangements for $L / D=1.5$. When $L / D=2.0$, 
although shear layers are also developed from the top and bottom side of the cylinder array in the inline and the staggered arrangements, they are weaker than their counterparts for $L / D=1.5$ because more fluid passes through instead of going around the cylinder array. The combination of the weakened shear layers and the strong jet-like flows through the gaps of cylinder rows prevent the interaction of the shear layers from the top and the bottom sides of the cylinder array from occurring in the immediate downstream of the cylinder array at $L / D=2$. The vortex shedding occurs at about $45 \mathrm{D}$ downstream from the centre of cylinder array for the inline square arrangement and about $30 \mathrm{D}$ for the staggered square arrangement. The occurrence of vortex shedding at a further downstream location in the inline arrangement than that in the staggered arrangement is because the gap flows in the former arrangement are considerably stronger that those in the latter. Although vortex shedding is observed behind both cylinder arrangements for $L / D=2$, both drag and lift oscillations are very weak (Fig. 5). This is because the vortex shedding occurs far away downstream from the cylinder array and the pressure variation induced by vortex shedding has little influence on pressure distribution on the cylinder surfaces. 

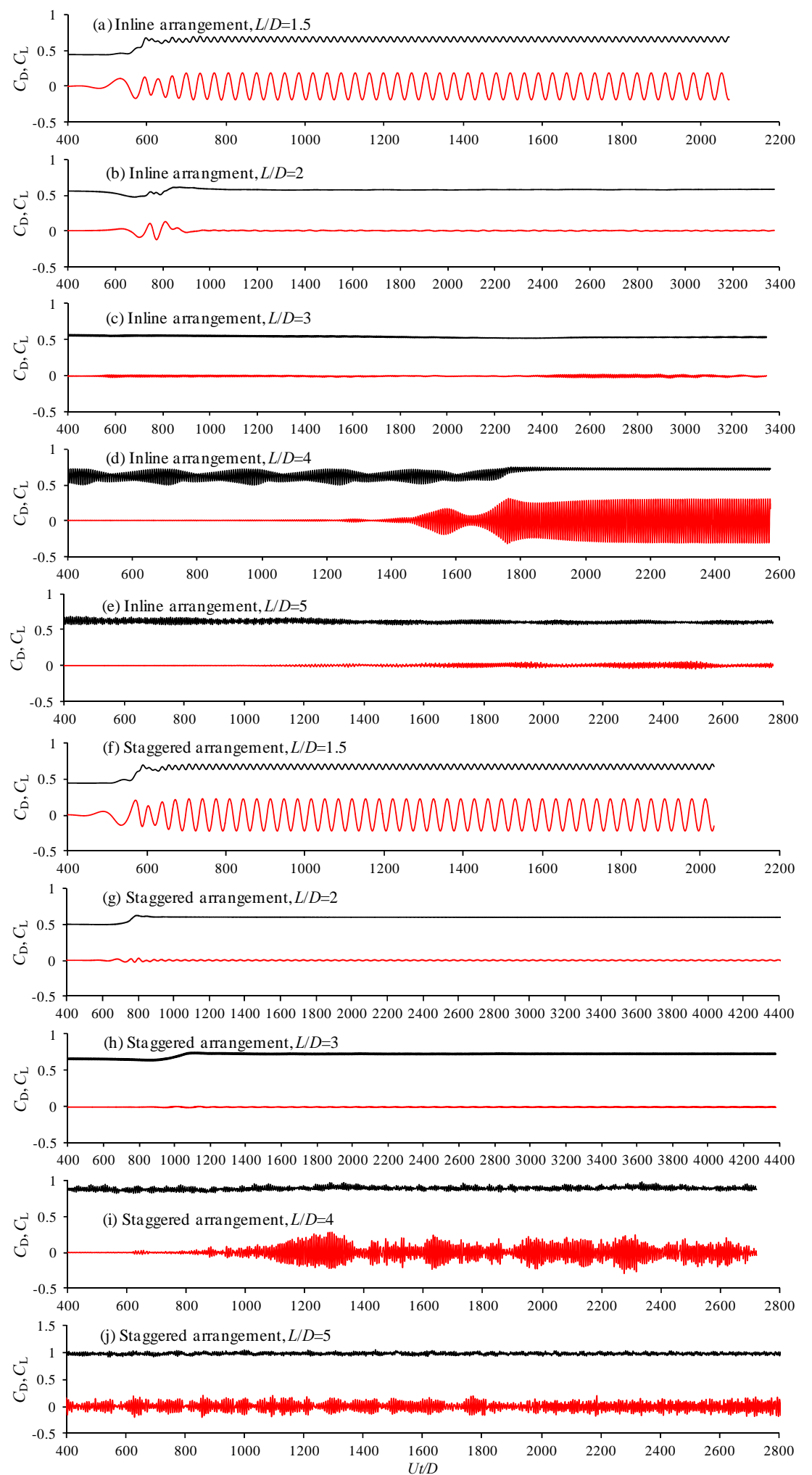

Fig. 5 Time history of the force coefficient for a stationary cylinder array. (a-e) Inline arrangement; (f-j) Staggered arrangement.

For the inline arrangement with $L / D=3$, vortex shedding occurs only behind the most downstream cylinders. The wake of the cylinder array in the inline square arrangement is 
characterised by a vortex street in the wake of each row of the cylinders rather than a single global vortex street as in the corresponding case of $L / D=1.5$ and 2.0. The wake behind the array of cylinders with the staggered arrangement for $L / D=3.0$ is somewhat similar to that behind the cylinder array in the inline square arrangement at $L / D=2.0$. The vortex shedding that occurs from only two cylinders at the bottom-left and the top-left corners appears not to have significant influence on the global flow. Vortex shedding from each individual cylinders occurs at the spacing ratios of $L / D=4$ and 5 . This is in consistent with the flow features observed around two tandem cylinders and four cylinders as reviewed in Section 1. The global vortex shedding around the cylinder array almost completely disappears due to the weakness of the shear layers from of the two sides of the cylinder array for $L / D=4$ and 5 . The vortex shedding patterns around rows of the cylinders in the inline arrangement for $L / D=4$ are largely in phase, resulting in regular and noticeable oscillations of both lift and drag coefficients as shown in Fig. 4 . The flow pattern for $L / D=4$ in the inline arrangement shown in Fig. 3 (d) shows an example of in-phase shedding pattern and the in-phase is especially obvious for the vortex shedding around the cylinders in the first three columns of cylinders. The vortex shedding around the cylinders in the array with the staggered arrangement for $L / D=4$ is largely in phase but not as obvious as in the inline arrangement except for the vortex shedding patterns around the six cylinders in the upstream column of the array, due to the strong interactions of vortex shedding processes from individual cylinders. The strong interaction among vortex shedding flows is evidenced by irregular but yet large oscillations of lift coefficient shown in Fig. 4 (i). In contrast, the vortex shedding patterns around the cylinders in the cylinder array with both arrangements for $L / D=5$ are largely anti-phase, resulting in very small oscillation amplitudes of both lift and drag coefficients as shown in Fig. 4 (e) and (j), although the anti-phase vortex shedding features are more pronounced in the inline square arrangement than in the staggered square arrangement. In the inline arrangement, the vortex shedding pattern for $L / D=4$ is in the anti-phase pattern until $U t / D=1400$ as evidenced by the zero lift coefficient as shown in Fig. 5 (a). The vortex shedding flow pattern starts to transit from 
an anti-phase to an in-phase pattern from $U t / D=1400$ to 1800 and remains an in-phase pattern for the rest of the simulation until $U t / D=2600$. At $L / D=5$, the vortex shedding flow is largely in an antiphase pattern for the whole simulation period $(U t / D=2800)$. The force coefficients for flow past the staggered square cylinder array at $L / D=4$ and 5 are generally irregular due to strong interactions among the vortex shedding processes from individual cylinders as shown in Fig. 4 (h) and (j).

\section{Flow-induced vibration of a square array of cylinders}

When flow-induced vibration of a square array of cylinders is simulated, only the inline arrangement is considered and the simulations are conducted at two spacing ratios: $L / D=2$ and 5 . Each cylinder is elastically mounted by a spring and vibrated independently as shown in Fig. 2. For the convenience of discussion, the cylinders are labelled as C1 - C36, respectively; the rows of the cylinders are labelled as rows 1 to 6 ; the columns of the cylinders are labelled as columns 1 to 6 . The proximity of the cylinders will make the vibration of each cylinder different from that of an isolated single cylinder. When the spacing ratio is $L / D=2$, the simulation cannot be conducted for reduced velocities greater than 16 because of strong distortion of the computational mesh. Because of this, the range of the simulated reduced velocity is $1-16$ for $L / D=2$ and $1-30$ for $L / D=30$.

Fig. 6 shows the contours of the nondimensional vorticity for VIV of the cylinder array for $L / D=2$. Fig. 7 shows the variation of the vibration amplitude with the reduced velocity. Only the amplitudes of cylinders $1-18$ (denoted by C1 - C18 hereafter) are shown in Fig. 7, because the amplitudes of top three rows of cylinders are found to be the same as those of the bottom three rows of cylinders, respectively. Different from that of stationary cylinders in Fig. 4 (b), two rows of vortices are aligned on the top and bottom sides in the wake of the cylinder in Fig. 6, instead of two free shear layers. The two rows of vortices are shed from the top and bottom rows of cylinders. When $V_{\mathrm{r}}=4$, the vortices are shed only from the gap between cylinders 1 and 2 (bottom left corner) and the gap between cylinder 31 and 32 (top left corner). Obvious vortex motion is observed behind 
the most upstream column of cylinders. The flow is almost static after the second upstream column of cylinders, leading to zero vibration amplitudes of cylinders in columns 3 to 6 as shown in Fig. 7. With increasing reduced velocity, the motion of the vortices extends towards downstream. When the reduced velocity is $V_{\mathrm{r}}=8$, the vortices are shed from the top and bottom cylinders of the first three columns and vortex motion extends to behind the third column of the cylinder. At this reduced velocity the flow behind the two downstream columns of cylinders (columns 5 and 6) is still almost steady, leading to zero amplitudes of the cylinders in columns 5 and 6 as shown in Fig. 7. Dynamic vortex motion is observed behind all the cylinders at $V_{\mathrm{r}}=12$ as shown in Fig. 6 (c). Correspondingly, all the cylinders have non-zero amplitudes at $V_{\mathrm{r}}=12$ as shown in Fig. 7. Obvious vortex shedding behind the 6 most downstream cylinders occurs at $V_{\mathrm{r}}=12$ and become stronger at $V_{\mathrm{r}}=16$.
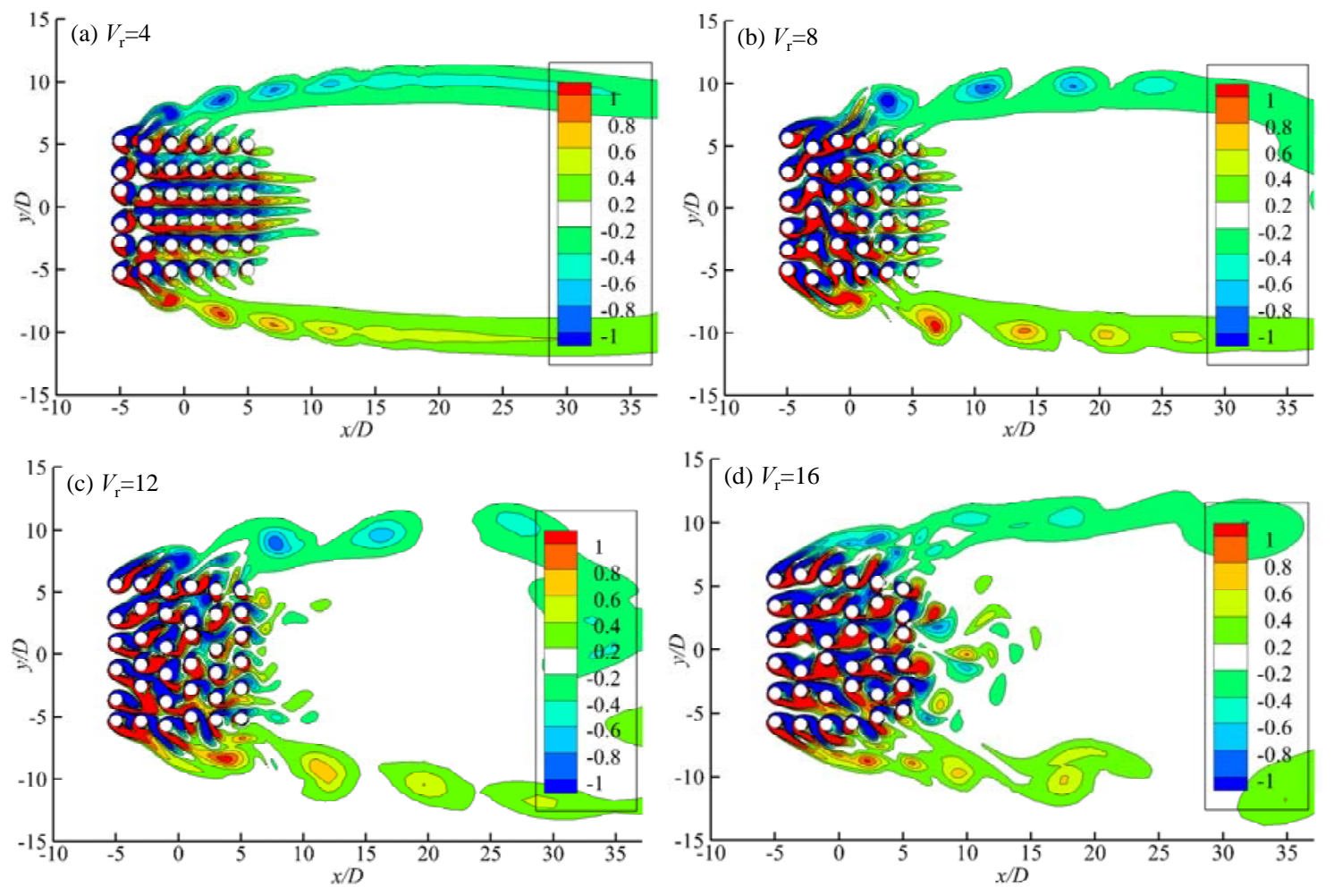

Fig. 6 Contours of the nondimensional vorticity for VIV of the cylinder array for $L / D=2$

It can be seen in Fig. 7 that the minimum reduced velocity for the vibration of cylinders 11 and 12, the most downstream two cylinders in the two middle rows, are the highest among other 
cylinders. This is because the influence of the vortex motion reaches these two cylinders the latest. Although the vibration amplitudes of the most downstream column of cylinders starts at higher reduced velocity, once the vibration starts, the amplitudes of these cylinders increase with increasing reduced velocity and are greater than those of the most upstream column of cylinders at $V_{\mathrm{r}}=16$.
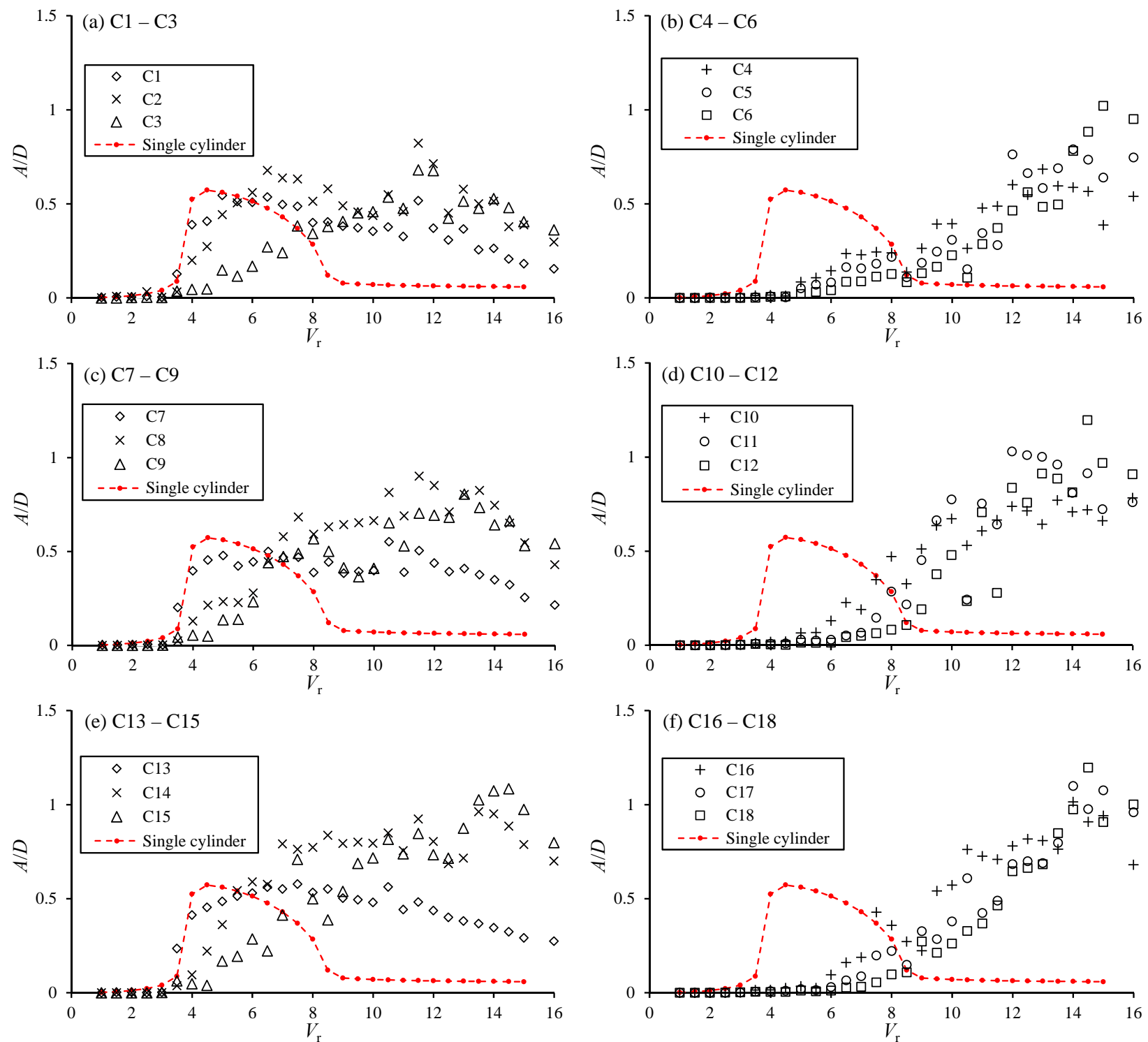

Fig. 7 Variation of the vibration amplitude with the reduced velocity for $L / D=2$

Fig. 8 shows the spectra of the displacement and the lift coefficient for $V_{\mathrm{r}}=5,9$ and 14 . The amplitudes of the displacement and the lift coefficient are normalized by their corresponding peak values, respectively. When $V_{\mathrm{r}}=5$, the response displacement and the lift coefficient of all the 
cylinders have the same predominant frequency. The very sharp spikes in Fig. 8 (a) indicate the regularity of the vibration. As the reduced velocity increases to 9, the lift coefficient of each of cylinders 1 to 3 has three dominant frequencies. The frequency of the displacement is the same as the lowest frequency of the lift coefficient, which is closest to the natural frequency. For $V_{\mathrm{r}}=9$, distinct peak frequencies can only be observed in the upstream cylinders C1-C3. When the reduced velocity is increased to 16, the vibrations of all cylinders become irregular.
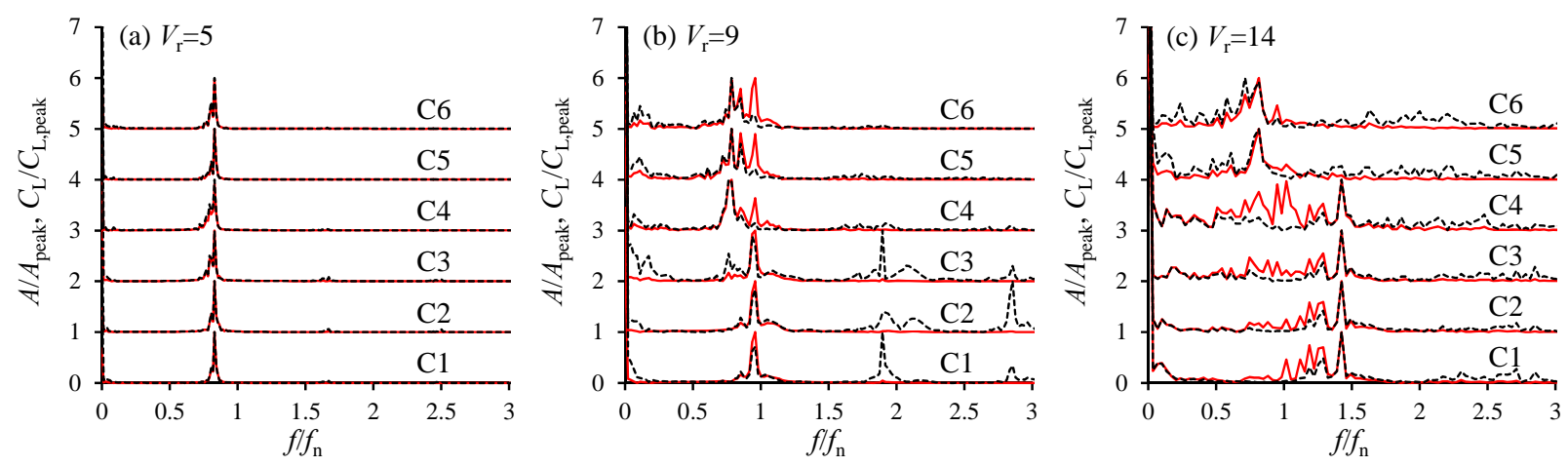

Fig. 8 FFT spectra of the displacement and the lift coefficient (normalized by the peak values) for $L / D=2$. Solid and the dashed curves are the FFT spectra of the displacement and the lift coefficient, respectively.

Fig. 9 shows the variation of the response frequency with the reduced velocity for $L / D=2$. The frequencies for $V_{\mathrm{r}} \leq 3$ are not shown because the flow is found to be steady as shown in Fig. 4 (b) and the vibration amplitudes are zero. It can be seen that the vibration frequencies of all the cylinders are smaller than that of a single cylinder, except at two reduced velocities of $V_{\mathrm{r}}=3.5$ and 4 . When the reduced velocity is less than 5.5 inclusive, the frequencies of all the cylinders are the same. When $V_{\mathrm{r}} \leq 5.5$, response is dominated by a single frequency as shown in Fig. 8 (a), because the vortex shedding only occurs from the most upstream columns of the cylinders, leading to weak vortex interaction. As the reduced velocity exceeds 6, the vortex shedding from more cylinders occurs and there is strong interaction between the vortices. It is interesting to see in Fig. 9 that the vibration 
frequencies of the upstream three columns of cylinders are generally higher than that of the downstream three columns of cylinders, with a few exceptions.
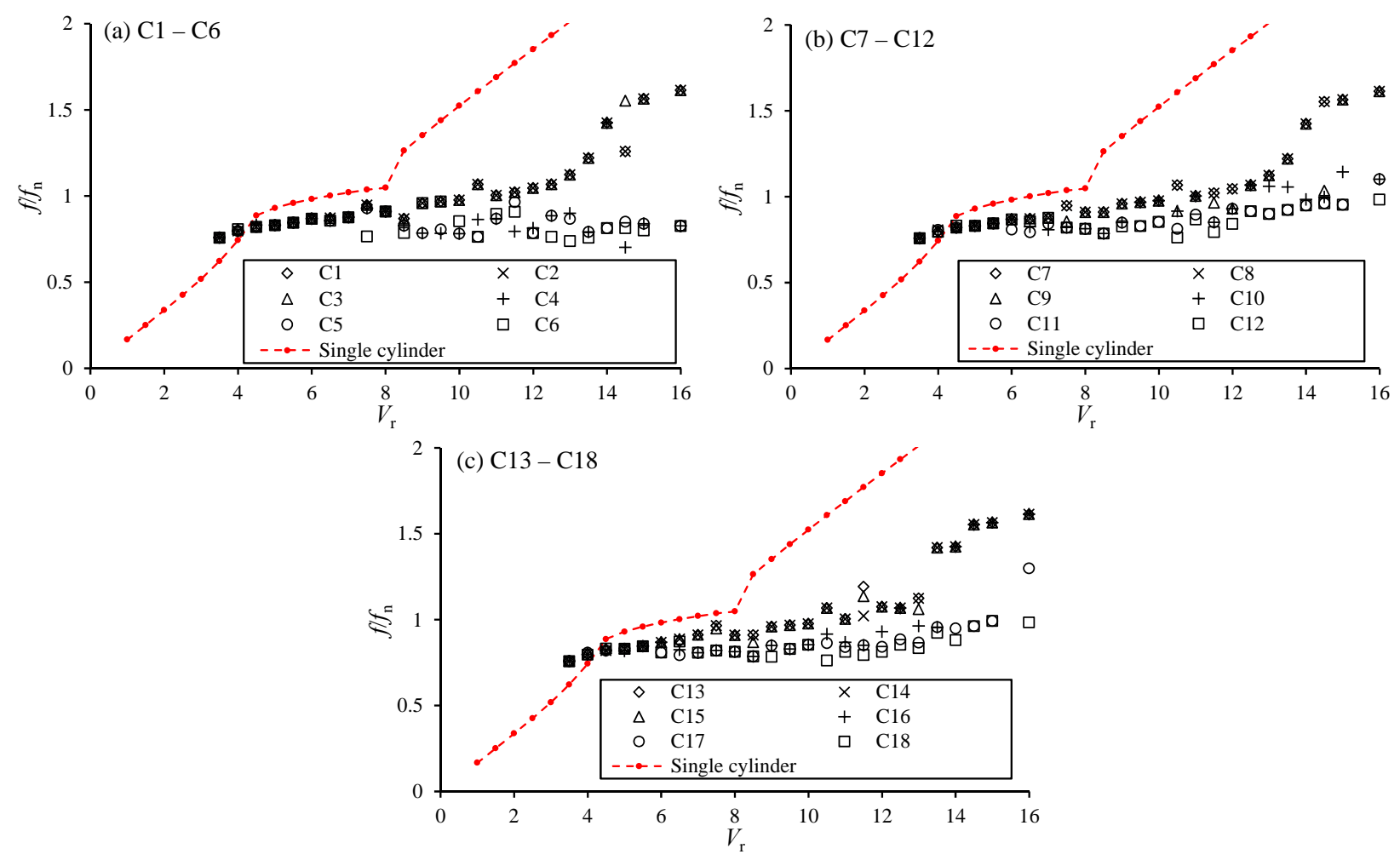

Fig. 9 Variation of the frequency of the vibration displacement with the reduced velocity for $L / D=2$.

Fig. 10 and Fig. 11 show the variations of the vibration amplitude and frequency with the reduced velocity, respectively for $L / D=5$. The response amplitudes of the cylinders in different columns are clearly different from each other. Because the spacing ratio has been very large, the response amplitude and frequency of the first column of cylinders are very close to their counterparts of a single cylinder, respectively. Due to the larger spacing ratio, the vibrations of the cylinders in different columns are more different from each other than those for the smaller spacing ratio of $L / D=2$. Assi et al. (2006) found that, when a cylinder is elastically mounted in the wake of a fixed cylinder, the response amplitude increases continuously with increasing reduced velocity until the reduced velocity is about 13 . Bao et al. (2011) found that the response amplitude of an elastically mounted cylinder in the wake of a fixed cylinder, maintains high amplitude after it peaks at a higher reduced velocity. In this study, it was found that reduced velocity where the response amplitude 
peaks increases with increasing column number. It can be seen in Fig. 10 that in each row of cylinders, the most downstream cylinder has the maximum response amplitude in the whole simulated reduced velocity range.
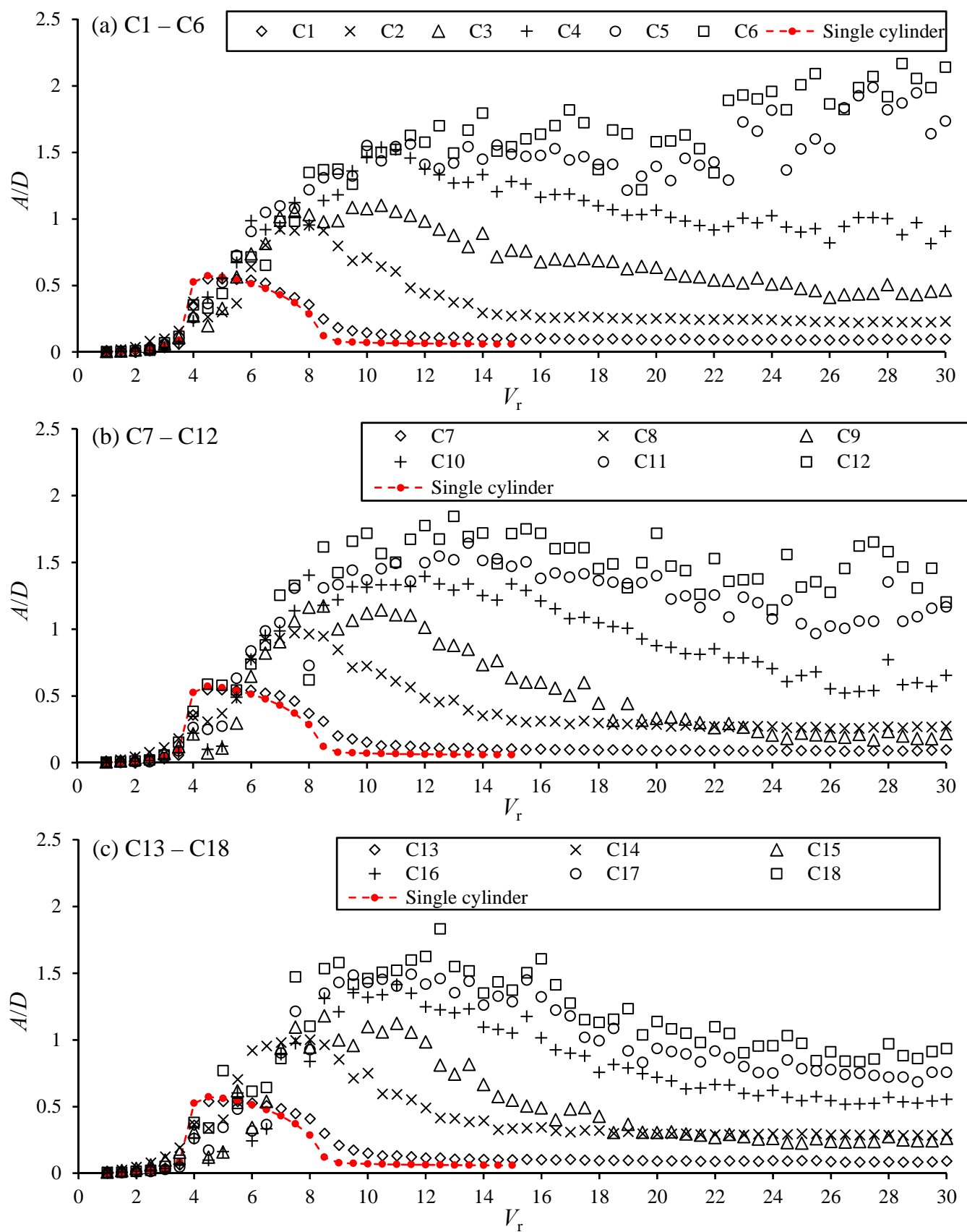

Fig. 10 Variation of the vibration amplitude with the reduced velocity for $L / D=5$ 

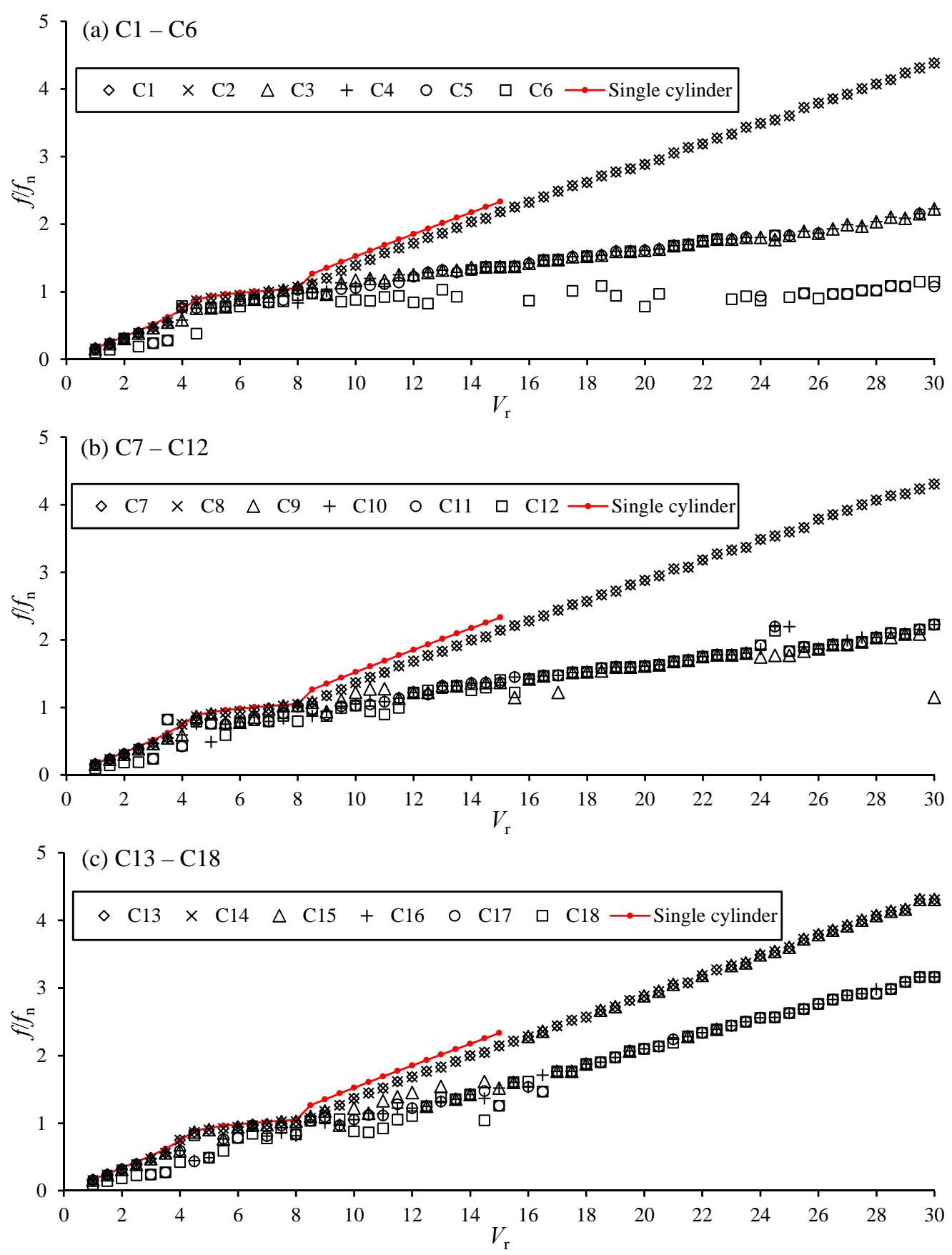

Fig. 11 Variation of the vibration frequency with the reduced velocity for $L / D=5$

It can be seen that the vibration frequencies of the first column of cylinders C1, C7 and C13 are the same as those of the second columns of cylinders C2, C8 and C14, respectively. The increase rates of the vibration frequency of the cylinders in columns 3 to 6 with increasing reduced velocity are reduced significantly compared with that of a single cylinder. Brika and Laneville (1999) attributed the reduction of the increase rate of the vortex shedding frequency of an elastically 
mounted cylinder in the wake of a fixed cylinder to the reduction of the mean flow velocity in the wake of the upstream cylinder. It can be seen in Fig. 11 that the response frequencies of the cylinders in the most downstream columns, especially those of the two bottom cylinders (C5 and C6) are reduced more than those in the middle columns.
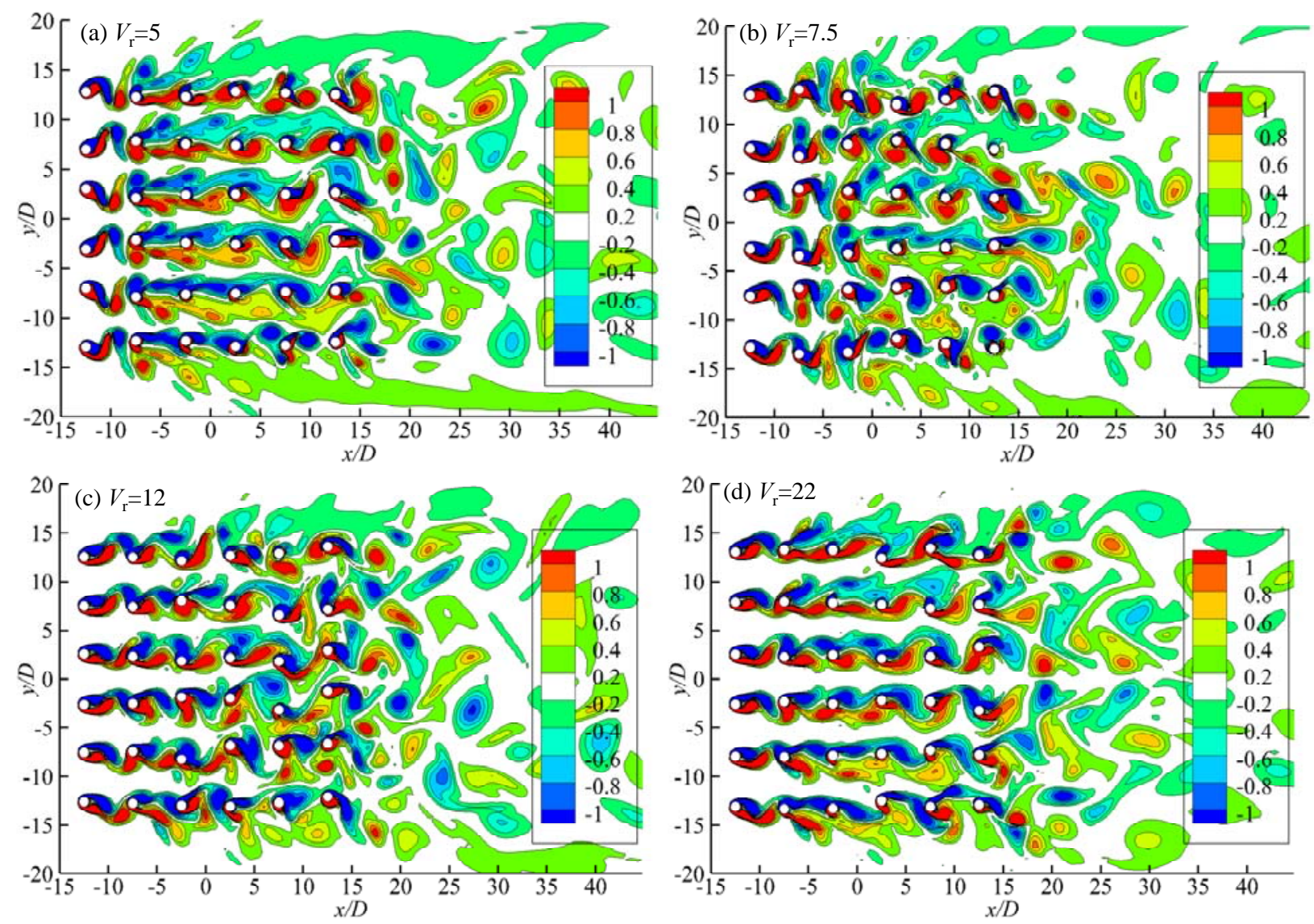

Fig. 12 Vorticity contours for $L / D=5$ at four selected reduced velocities

Fig. 12 shows the contours of the vorticity for $L / D=5$ and four reduced velocities. The vortex shedding pattern of the most upstream column of cylinders are similar to that of a single cylinder, due to large spacing ratio. The flow patterns are in a symmetric pattern around the two upstream columns of cylinders for all the reduced velocities. When $V_{\mathrm{r}}=7.5$ and 12 , the vortex flow around cylinders in columns $4-6$ becomes very irregular, leading to broad-banded lift coefficient as shown in the FFT spectra in Fig. 13 (b). When the reduced velocity is increased to $\underline{22}$, the vortex shedding 


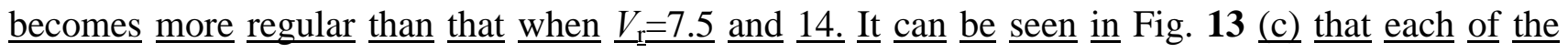

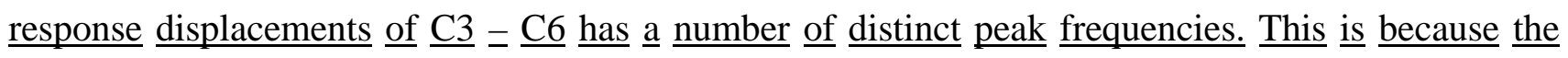

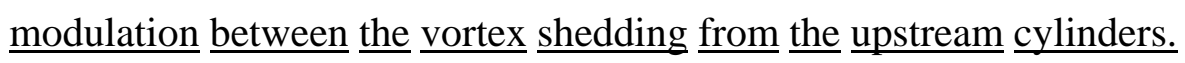
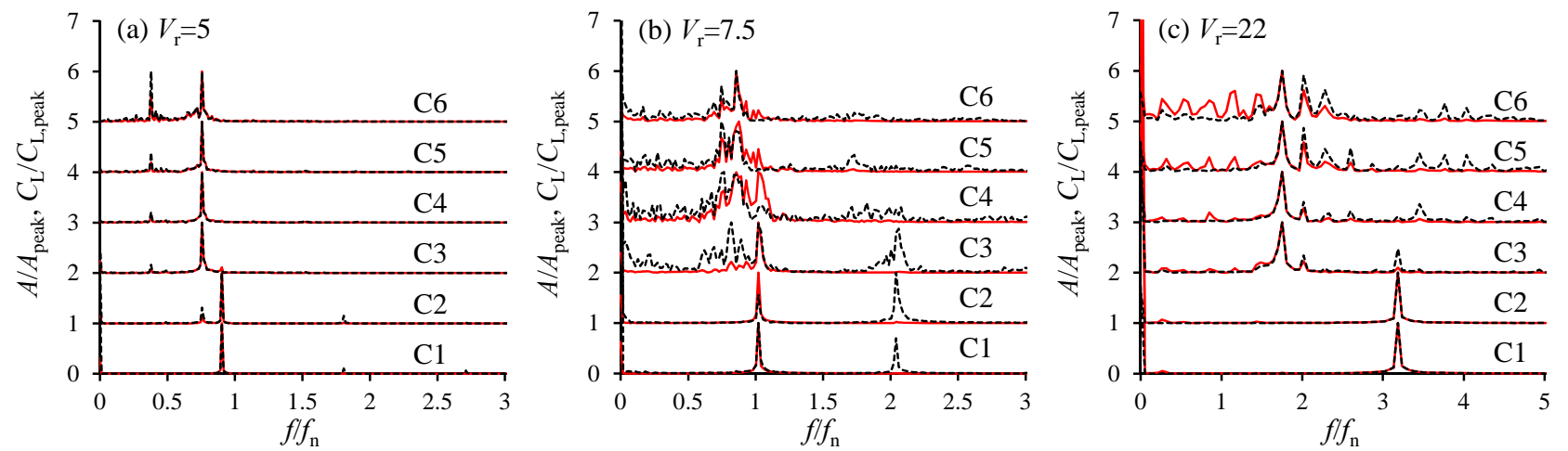

Fig. 13 FFT spectra of the displacement (solid curves) and the lift coefficient (dashed curves) (normalized by the peak values) for $L / D=5$.

\section{Conclusions}

Flow around and flow-induced vibration of a square array of cylinders are investigated by twodimensional numerical simulations. Flow past 36 cylinders in an inline arranged square array and flow past 33 cylinders in a staggered arranged square array is firstly simulated for $\operatorname{Re}=100$ and the spacing ratios of $L / D=1.5,2,3,4,5$. Only one vortex shedding street is observed in the wake of the cylinder array at small spacing ratios $(L / D=1.5$ for the inline square arrangement and $L / D=1.5$ and 2 for the staggered square arrangement). It appears the critical spacing ratio for the single vortex street of the staggered square arrangement is higher than that of the inline square arrangement. At intermediate spacing ratios ( $L / D=3$ for both arrangements), vortex shedding is suppressed both from individual cylinders and from the cylinder array, resulting in very small lift coefficient. As the spacing ratio further increases ( $L / D \geq 4$ ), vortex shedding from each individual cylinder in the array occurs. Both anti-phase and in-phase vortex shedding patterns are observed and the anti-phase vortex shedding leads to a near zero lift force on the cylinder system. 
Flow-induced vibration of 36 cylinders in an inline square array is simulated for a constant Reynolds number of 100, a constant mass ratio of 2.5, two spacing ratios of $L / D=2$ and 5 and a wide range of reduced velocities. When $L / D=2$, the flow is almost static downstream column-2 cylinders, leading to zero vibration amplitudes of the cylinders in columns 3 to 6 until $V_{\mathrm{r}}=4.5$. The dynamic vortex flow progresses towards downstream cylinders with increasing reduced velocities. When the spacing ratio is $L / D=5$, the response amplitudes and frequencies of the cylinders in the most upstream column are very similar to that of a single cylinder. The response amplitudes of the cylinders in the downstream columns in the array peak at higher reduced velocity and the maximum amplitude increases. While the response frequencies of the cylinders in columns 1 and 2 are slightly smaller than that of a single cylinder, the increasing rates of the response frequency of the cylinders in columns 3 to 6 are much smaller than that of a single cylinder and the increase rate reduces with increasing column number.

\section{Acknowledgment}

The authors would like to acknowledge the support from Australia Research Council through ARC Discovery Projects Program (Grant No. DP150104644). The calculations were carried out on the Computational Facilities of Intersect Australia Ltd. in NSW, Australia.

\section{References}

Alam, M. M., Moriya, M., Sakamoto, H., 2003. Aerodynamic characteristics of two side-by-side circular cylinders and application of wavelet analysis on the switching phenomenon. Journal of Fluids and Structures 18, 325-346.

Anagnostopoulos, P., Bearman, P.W., 1992. Response characteristics of a vortex-excited cylinder at low Reynolds numbers. Journal of Fluids and Structures 6, 39-50.

Assi, G.R.S., Meneghini, J.R., Aranha, J.A.P., Bearman, P.W., Casaprinma, E., 2006. Experimental investigation of flow-induced vibration interference between two circular cylinders. Journal of Fluids and Structures 22, 819-827.

Ball, D.J., Hall, C.D., 1980. Drag of yawed pipe groups at low Reynolds numbers. Journal of the waterway Port Coastal and Ocean Division 106 (2), 229-238. 
Bao, Y., Zhou, D., Tu, J., 2011. Flow interference between a stationary cylinder and an elastically mounted cylinder arranged in proximity. Journal of Fluids and Structures 27, 1425-1446.

Bearman, P.W., Wadcock, A.J., 1973. The interaction between a pair of circular cylinders normal to a stream. Journal of Fluid Mechanics 61, 499-511.

Blevins, R., 2001. Flow-Induced Vibration, second ed., Krieger Publishing Company.

Bokaian, A., Geoola, F., 1984. Wake-induced galloping of two interfering circular cylinders. Journal of Fluid Mechanics 146, 383-415.

Brika, D., Laneville, A., 1999. The flow interaction between a stationary cylinder and a downstream flexible cylinder. Journal of Fluids and Structures 13 (5), 579-606.

Dehkordi, B.G., Jafari, H.H., 2009. Numerical simulation of flow through tube bundles in in-line square and general staggered arrangements. International Journal of Numerical Methods for Heat \& Fluid Flow 19 (8), 1038-1062.

Eames, I., Hunt, J.C.R., Belcher, S.E., 2004. Inviscid mean flow through and around groups of bodies. Journal of Fluid Mechanics 515, 371-389.

Farrant, T., Tan, M., Price, W.G., 2000. A cell boundary element method applied to laminar vortexshedding from arrays of cylinders in various arrangements. Journal of Fluids and Structures 14, 375-402.

Feng, C.C., 1968. The Measurements of Vortex-induced Effects in Flow Past Stationary and Oscillating Circular and D-section Cylinder. Master's Thesis. Universityof British Columbia, Vancouver, BC, Canada.

Fontaine, E., Morel, J., Scolan, Y., Rippol, T., 2006. Riser interference and VIV amplification in tandem configuration. International Journal of Offshore and Polar Engineering 16 (1), 33-40.

Govardhan, R., Williamson, C.H.K., 2000. Modes of vortex formation and frequency response of a freely vibrating cylinder. Journal of Fluid Mechanics 420, 85-130.

Guilmineau, E., Queutey, P., 2004. Numerical simulation of vortex-induced vibration of a circular cylinder with low mass-damping in a turbulent flow. Journal of Fluids and Structures 19, 449466.

Han, Z., Zou, D., Gui, X., Tu, J., 2013. Numerical study of flow past four square-arranged cylinders using spectral element method. Computers \& Fluids 84, 100-112.

Huera-Huarte, F.J., Gharib, M., 2011. Flow-induced vibrations of a side-by-side arrangement of two flexible circular cylinders. Journal of Fluids and Structures 27, 354-366.

Jauvtis, N., Williamson, C.H.K., 2004. The effect of two degrees of freedom on vortex-induced vibration at low mass and damping. Journal of Fluid Mechanics 509, $23-62$.

Kataoka, H., 2008. Numerical simulations of a wind-induced vibrating square cylinder within turbulent boundary layer. Journal of Wind Engineering and Industrial Aerodynamics 96, 19851997.

Kevlahan, N.K.-R., 2011. The role of vortex wake dynamics in the flow-induced vibration of tube arrays. Journal of Fluids and Structures 27, 829-837.

Kevlahan, N.K.-R., Wadsley, J., 2005. Suppression of three-dimensional flow instabilities in tube bundles. Journal of Fluids and Structures 20, 611-620.

Khalak, A., Williamson, C.H.K., 1996. Dynamics of a hydroelastic cylinder with very low mass and damping. Journal of Fluids and Structures 10, 455-472. 
Khalak, A., Williamson, C.H.K., 1997. Fluid forces and dynamics of a hydroelastic structure with very low and damping. Journal of Fluids and Structures 11, 973-982.

Khalak, A., Williamson, C.H.K., 1999. Motions, forces and mode transitions in vortex-induced vibrations at low mass-damping. Journal of Fluids and Structures 13, 813-851.

Kim, H.J., Durbin, P.A., 1988. Investigation of the flow between a pair of cylinders in the flopping regime, Journal of Fluid Mechanics, 196, 431-448.

Lam, K., Fang, X., 1995. The effect of interference of four equispaced cylinders in cross flow on pressure and force coefficients. Journal of Fluids and Structures 9, 195-214.

Lam, K., Li, J.Y., Chan, K.T., So, R.M.S., 2003a. Flow pattern and velocity field distribution of cross-flow around four cylinders in a square configuration at low Reynolds number. Journal of Fluids and Structures 17, 665-679.

Lam, K., Li, J.Y., So, R.M.C., 2003b. Force coefficients and Strouhal numbers of four cylinders in cross flow. Journal of Fluids and Structures 18, 305-324.

Lam, K., Lo, S.C., 1992. A visualization study of cross-flow around four cylinders in a square configuration. Journal of Fluids and Structures 6, 109-131.

Lam, K., Zou, L., 2009. Experimental study and large eddy simulation for the urbulent flow around four cylinders in an in-line square configuration. International Journal of Heat and Fluid Flow 30, 276-285.

Lam, K., Zou, L., 2010. Three-dimensional numerical simulation of cross-flow around four cylinders in an in-line square configuration. Journal of Fluids and Structures 26, 482-502.

Leontini, J.S., Thompson, M.C., Hourigan, K., 2006. The beginning of branching behaviour of vortex-induced vibration during two-dimensional flow. Journal of Fluids and Structures 22, 857864.

Longatte, E., Bendjeddou, Z., Souli, M., 2003. Methods for numerical study of tube bundle vibrations in cross-flows. Journal of Fluids and Structures 18, 513-528.

Meneghini, J.R., Saltara, F., Siqueira, C.L.R., Ferrari, J.J.A., 2001. Numerical simulation of flow interference between two circular cylinders in tandem and side-by-side arrangements. Journal of Fluids and Structures 15 (2), 327-350.

Mittal, S., Kumar, V., 1999. Finite element study of vortex-induced cross-flow and in-line oscillations of a circular cylinder at low Reynolds numbers. International Journal for Numerical Methods in Fluids 31, 1087-1120.

Mittal, S., Kumar, V., 2001. Flow-induced oscillations of two cylinders in tandem and staggered arrangements. Journal of Fluids and Structures 15 (5), 717-736.

Mittal, S., Kumar, V., 2004. Vortex induced vibrations of a pair of cylinders at Reynolds number 1000. International Journal of Computational Fluid Dynamics 18, 601-614.

Mizushima, J., Suehiro, N. 2005. Instability and transition of flow past two tandem circular cylinders. Physics of Fluids 17 (10), 104107.

Nicolle, A., Eames, I., 2011. Numerical study of flow through and around a circular array of cylinders. Journal of Fluid Mechanics 679, 1-31.

Pan, Z.Y., Cui, W.C., Miao, Q.M., 2007. Numerical simulation of vortex-induced vibration of a circular cylinder at low mass-damping using RANS code. Journal of Fluids and Structures 23, 23-37. 
Prasanth, T.K., Behara, S., Singh, S.P., Kumar, R., Mittal, S., 2006. Effect of blockage on vortexinduced vibrations at low Reynolds numbers. Journal of Fluids and Structures 22, 865-876.

Prasanth, T.K., Mittal, S., 2008. Effect of blockage on free vibration of a circular cylinder at low Re. international journal of numerical methods in fluids 58, 1063-1080.

Price, S.J., Paidoussis, M.P., Mark, B., 1995. Flow visualization of the interstitial cross-flow through parallel triangular and rotated square arrays of cylinders. Journal of Sound and Vibration 181, 85-98.

Rahmanian, M., Zhao, M., Cheng, L., Zhou, T., 2012. Two-degree-of-freedom vortex-induced vibration of two mechanically coupled cylinders of different diameters in steady current. Journal of Fluids and Structures 35, 133-159.

Rahmanian, M., Cheng, L., Zhao, M., Zhou, T., 2014. Lock-in study of two side-by-side cylinders of different diameters in close proximity in steady flow. Journal of Fluids and Structures 49, 386-411.

Ruscheweyh, H.P. 1983. Aeroelastic interference effects between slender structures. Journal of Wind Engineering and Industrial Aerodynamics. 14 (1-3), 129-140.

Sayers, A.T., 1988. Flow interference between four equispaced cylinders when subjected to a cross flow. Journal of Wind Engineering and Industrial aerodynamics 31, 9-28.

Sayers, A.T., 1990. Vortex shedding from groups of three and four equispaced cylinders situated in a cross flow. Journal of Wind Engineering and Industrial Aerodynamics 34, 213-221.

Singh, S.P., Mittal, S., 2005. Vortex-induced oscillations at low Reynolds numbers: Hysteresis and vortex-shedding modes. Journal of Fluids and Structures 20, 1085-1104.

Sumner, D., 2010. Two circular cylinders in cross-flow: A review. Journal of Fluids and Structures 26, 849-899.

Sweeney, C., Meskell, C., 2003. Fast numerical simulation of vortex shedding in tube arrays using a discrete vortex method. Journal of Fluids and Structures 18, 501-512.

Tasaka, Y., Kon, S., Schouveiler, L., Gal, P.L., 2006. Hysteretic mode exchange in the wake of two circular cylinders in tandem. Physics of Fluids 18, 084101.

Tong, F., Cheng, L., Zhao, M., Zhou, T., Chen, X., 2014. The vortex shedding around four circular cylinders in an in-line square configuration. Physics of Fluids 26, 024112.

Tong, F., Cheng, L., Zhao, M., 2015. Three-dimensional numerical simulations of steady flow past two cylinders in staggered arrangements. Journal of Fluid Mechanics 765, 114-149.

Williamson, C.H.K., 1985. Evolution of a single wake behind a pair of bluff bodies. Journal of Fluid Mechanics 159, 1-18.

Williamson, C.H.K., 1996. Vortex dynamics in the cylinder wake. Annual Review of Fluid Mechanics 28, 477-539.

Yu, L.H., Zhan, J.M., Li, Y.S., 2013. Numerical investigation of drag force on flow through circular array of cylinders. Journal of Hydrodynamics 25 (3), 330-338.

Zang, Z., Gao, F., Cui, J.S., 2013, Physical modeling and swirling strength analysis of vortex shedding from near-bed piggyback pipelines. Applied Ocean Research 40, 50-59.

Zhao, M., 2013. Flow induced vibration of two rigidly coupled circular cylinders in tandem and sideby-side arrangements at a low Reynolds number of 150. Physics of Fluids. 25, 123601. 
Zhao, M., Cheng, L., 2011. Numerical simulation of two-degree-of-freedom vortex-induced vibration of a circular cylinder close to a plane boundary. Journal of Fluids and Structures 27, 1097-1110.

Zhao, M, Cheng, L., 2012. Numerical simulation of vortex-induced vibration of four circular cylinders in a square configuration. Journal of Fluids and Structures 31, 125-140.

Zhao, M., Cheng, L., 2014. Vortex-induced vibration of a circular cylinder of finite length. Physics of Fluids, 26, 015111.

Zhao, M., Cheng, L., An, H., Lu, L., 2014. Three-dimensional numerical simulation of vortexinduced vibration of an elastically mounted rigid circular cylinder in steady current, Journal of Fluids and Structures 50, 292-311.

Zhao, M., Cheng, L., Zhou, T., 2013. Numerical simulation of vortex-induced vibration of a square cylinder at a low Reynolds number. Physics of Fluids 25, 023603.

Zhao, M., Yan, G., 2013. Numerical simulation of vortex-induced vibration of two circular cylinders of different diameters at low Reynolds number. Physics of Fluids 25, 083601. 


\section{List of Figures}

Fig. 1 Inline and staggered configurations of a square array of stationary cylinders in a fluid flow. (a) Inline arrangement; (b) Staggered arrangement; (c) Computational domain.

Fig. 2 Sketch of elastically supported an array of cylinders in an inline square arrangement

Fig. 3 Computational mesh around the cylinders. (a) $L=3$, Inline arrangement; (b) $L=1.5$, staggered arrangement

Fig. 4 Contours of the nondimensional vorticity for flow past a fixed cylinder array

Fig. 5 Time history of the force coefficient for a stationary cylinder array. (a-e) Inline arrangement;

(f-j) Staggered arrangement.

Fig. 6 Contours of the nondimensional vorticity for VIV of the cylinder array for $L / D=2$

Fig. 7 Variation of the vibration amplitude with the reduced velocity for $L / D=2$

Fig. 8 FFT spectra of the displacement and the lift coefficient (normalized by the peak values) for $L / D=2$. Solid and the dashed curves are the FFT spectra of the displacement and the lift coefficient, respectively.

Fig. 9 Variation of the frequency of the vibration displacement with the reduced velocity for $L / D=2$.

Fig. 10 Variation of the vibration amplitude with the reduced velocity for $L / D=5$

Fig. 11 Variation of the vibration frequency with the reduced velocity for $L / D=5$

Fig. 12 Vorticity contours for $L / D=5$ at four selected reduced velocities

Fig. 13 FFT spectra of the displacement (solid curves) and the lift coefficient (dashed curves) (normalized by the peak values) for $L / D=5$.

Fig. 14 Variation of the response amplitude with the reduced velocity

Fig. 15 Variation of the response frequency with the reduced velocity

Fig. 16 Time histories of the vibration displacement in the cross-flow direction

Fig. 17 Nondimensional vorticity contours for the vibrating cylinder arrays

Fig. 18 Phase plots of $C_{\mathrm{L}}$ versus $Y / D$

Fig. 19 Variation of the root mean square lift coefficient with the reduced velocity. (a) $L / D=1.5$ and 2; (b) $L / D=3$ and 5. 